\title{
EGF domain swap converts a D rosophila EGF receptor activator into an inhibitor
}

\author{
Bruce Schnepp, ${ }^{1,3}$ Timothy Donaldson, 1,3 \\ Gary Grumbling, ${ }^{1}$ Stephen Ostrowski, 1 \\ Ronen Schweitzer, ${ }^{2,4}$ Ben-Zion Shilo, \\ and Amanda Simcox ${ }^{1,5}$
}

\begin{abstract}
${ }^{1}$ Department of Molecular Genetics, The Ohio State University, Columbus, Ohio 43210 USA; ${ }^{2}$ Department of Molecular Genetics, Weizmann Institute of Science, Rehovet 76100, Israel
\end{abstract}

In D rosophila the function of the epidermal growth factor (EGF) receptor is modulated zygotically by three EGF-like proteins: Spitz (Spi), which is a potent activa tor; Vein (Nn), which is a moderate activator; and Argos (AOs), which is an inhibitor. Chimeric molecules were constructed in which the EGF domain of Vn was swapped with the EGF domain from each factor. The modified Vn proteins behaved both in vitro and in vivo with properties characteristic of the factor from which the EGF domain was derived. These results demonstrate that the EGF domain is the key determinant that gives DER inhibitors and activators their distinct properties.

Received January 13, 1998; revised version accepted February 10, 1998.

The Drosophila epi dermal growth factor (EGF) receptor (DER) is a member of the ErbB family of receptor tyrosine kinases (RTKs) and, like its vertebrate counterparts, controls cell differentiation, survival, and proliferation in many tissues throughout development (Perrimon and Perkins 1997; Schweitzer and Shilo 1997). DER function is modulated by four candidate ligands each of which possesses a predicted EGF-like domain. Gurken (Grk) and Spitz (Spi) are TGF $\alpha$-like proteins (Rutledge et al. 1992; N euman-Si lberberg and Schüpbach 1993). grk is a maternally active gene involved in establishing egg polarity (N euman-Silberberg and Schüpbach 1993; González-Reyes et al. 1995), whereas spi is zygotically active and functions in the embryo, adult eye, and wing (Rutledge et al. 1992; Freeman 1994b). Vn resembles the vertebrate neuregulins in that both possess an Ig-C2 domain in addition to the EGF-like domain (Schnepp et al. 1996). Vein $(V n)$ functions zygotically in the embryo and the adult wing (Schnepp et al. 1996; Simcox et al. 1996; Simcox 1997; Yarnitzky et al. 1997). Here we show that Vn is a moderate activator of DER signaling in comparison

[Key Words: Drosophila; vein; spitz; argos; EGF receptor; EGF] ${ }^{3} T h e s e$ authors contributed equally to this work.

4Present address: Department of Genetics, Harvard Medical School, Boston, Massachusetts 02115 USA.

${ }^{5}$ Corresponding author.

E-MAIL simcox.1@osu.edu; FAX (614) 292-4466. with Spi, which is a potent DER activator. Argos (Aos) has been recognized recently as an inhibitor of the DER pathway and was the first extracellular factor shown to inhibit an RTK in vivo (Schweitzer et al. 1995a). Aos functions in the embryo, adult eye, and wing (Freeman et al. 1992; Sawamoto et al. 1994; Golembo et al. 1996).

Considerable effort has been made to understand the structure-function relationships of vertebrate EGF-like mitogens to aid in the development of ErbB receptor superagonists or antagonists; however, to date these studies have not led to the design of effective factors (Groenen et al. 1994). The fly system offers a unique opportunity to define the mol ecular basis for the distinct properties of three natural ligands with different effects on a receptor and could facilitate the development of vertebrate factors with similar relative properties.

Spi, Vn, and Aos are structurally unrelated except within the EGF domain (Fig. 1A). The EGF domain contains a series of six cysteines, which form three di sulfide bonds to generate a looped structure, and a number of other highly conserved residues that are known to be required for binding and activating members of the vertebrate ErbB receptor family (Groenen et al. 1994). The EGF domains of $\mathrm{Vn}$ and Spi are not highly related (38\% conserved) but have more sequence conservation with each other than with Aos (Fig. 1A). Additionally, the length of the predicted $B$ loop that forms from the region between cysteines 3 and 4 is significantly longer in Aos than in the activating ligands (Fig. 1A). The low level of sequence homology and the structural differences in the EGF domain could account for the different effects that the proteins have on DER signaling. To determine whether the EGF domain is sufficient to confer these distinct properties we generated chimeric molecules by exchanging the EGF domain of $\mathrm{Vn}$ for those of Spi or Aos (Fig. 1A). The activity of these chimeras was compared with the native factors in vitro and in vivo.

\section{Results and Discussion}

Activation of DER pathway by $\mathrm{Vn}$ in vitro

Upon ligand binding, ErbB receptors dimerize, crossphosphorylate on carboxy-terminal tyrosine residues, and transmit a signal to the nucleus through the Ras/ Raf/ERK pathway (Egan and Weinberg 1993). Thus, DER tyrosine phosphorylation is the initial indication of pathway activation, and ERK phosphorylation is a result of the signal transduction. Secreted Spi (sSpi, the active form of Spi) and Aos have been shown to increase or decrease, respectively, the level of DER tyrosine phosphorylation in Drosophila S2-DER tissue-culture cells (Schweitzer et al. 1995a,b). We applied Vn produced by transfected S2 tissue-culture cells to S2-DER cells and showed that $\mathrm{Vn}$ is a DER activator and induced DER tyrosine phosphorylation in a dose-dependent fashion with a concomitant rise in ERK activation (Fig. 1B). These in vitro results provide bi ochemical evidence that the newly discovered $V n$ protein, which had been linked 
A

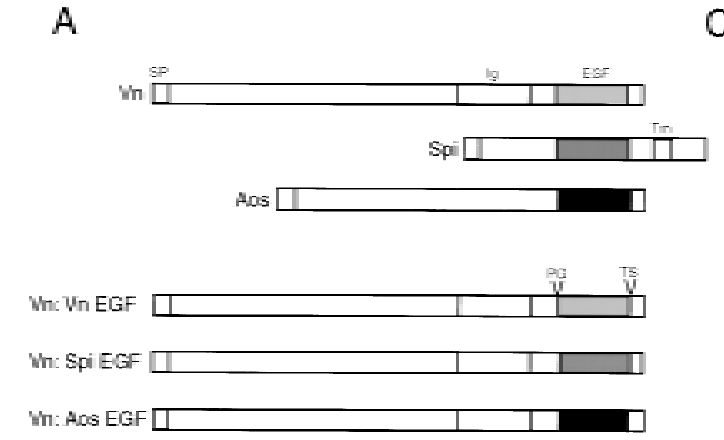

$\mathrm{C}$

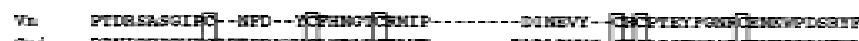

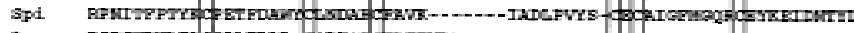

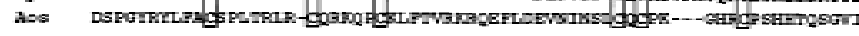

B

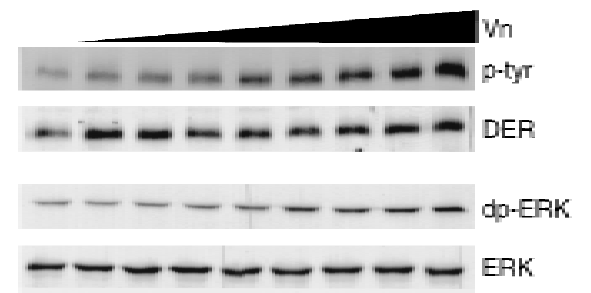

D

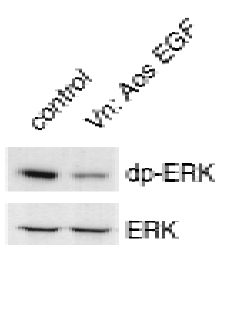

Figure 1. (A) Schematic of native and chimeric EGF-like proteins. The domain structure of native $\mathrm{Vn}, \mathrm{Spi}$, Aos, and chimeric proteins constructed between $\mathrm{Vn}$ and the $\mathrm{Vn}$, Spi, or Aos EGF domains is shown at the top. The manipulation to produce the chimeras results in the addition of 4 residues flanking the EGF domain in each chimera (shown only in the Vn:Vn EGF cartoon). (SP) Signal peptide: (Ig) immunoglobulin-like domain; (EGF) EGF-like domain; (TM ) transmembrane region. The al ignment of the EGF domains of $\mathrm{Vn}, \mathrm{Spi}$, and Aos is shown below. The six conserved cysteines are boxed. The spacing between cysteines 3 and 4 is significantly longer in Aos than any of the other proteins. (B) Activation of DER signaling by $V n$ in vitro. S2 cells expressing DER were exposed to increasing concentrations of $\mathrm{Vn}$ in conditioned medium. DER activation as measured by tyrosine phosphorylation (anti-p-tyr) increased relative to DER protein level (anti-DER) as $\mathrm{Vn}$ concentration increased. The level of ERK phosphorylation (anti-dp-ERK) also increased relative to ERK protein level (anti-ERK) as $\mathrm{Vn}$ concentration increased. The maximum level tested was a $60 \times$ concentration of $\mathrm{Vn}$-conditioned medium, and the preceding lanes are dilutions that differ by 0.125 increments. (C) Activation of DER signaling by sSpi, Vn, and the chimeras. S2-DER-expressing cells were exposed to control medium and medium conditioned with sSpi, Vn,Vn:Vn EGF, and Vn:Spi EGF. Control cells $(\rightarrow$ show that the basal level of DER tyrosine phosphorylation is low relative to DER protein level (anti-DER). SSpi-conditioned medium results in an elevation of DER phosphorylation (anti-p-tyr). Native $\mathrm{Vn}$ and the $\mathrm{Vn}: \mathrm{Vn}$ EGF chimera result in a modest elevation of DER tyrosine phosphorylation, whereas, the Vn-Spi:EGF chimera results in a high level of DER tyrosine phosphorylation. Corresponding relative increases in ERK activation (anti-dp-ERK) were seen with the factors. Similar levels of $\mathrm{Vn}$ and the $\mathrm{Vn}$ chimeras were added to the S2-DER cells as determined by Western analysis with anti$\mathrm{Vn}$. This concentration was equival ent to the highest used in the dose-response-experiment (B). (D) Vn:Aos EGF inhibits ligand-independent ERK activation. S2-DER cells exhibiting a high level of ligand-independent DER activation al so had high levels of ERK activation (anti-dp-ERK) relative to ERK protein level (anti-ERK). Addition of $\mathrm{Vn}$ :Aos EGF results in a lowering of the level of ERK activation.

to the pathway genetically (Schnepp et al. 1996; Yarnitzky et al. 1997), is a DER ligand. A di rect comparison of the potency of native $\mathrm{Vn}$ and sSpi in vitro cannot be made because the proteins have not been purified and the absolute levels of each protein in the media are thus unknown; however, we infer that sSpi is the more potent factor because the $\mathrm{Vn}$ :Spi EGF chimera has stronger ac- tivity than $V n$ and because sSpi is more potent than $\mathrm{Vn}$ in vivo (see below).

In vitro activity of $\mathrm{V} n$ chimeras resembles the factor from which the EGF domain derives

The level of activation of the chimeric proteins was monitored. The $\mathrm{Vn}: \mathrm{Vn}$ EGF chimera, which serves as a control for the effect of the additional residues introduced during construction of the chimeras, behaved like native Vn (Fig. 1C). In contrast, possession of the Spi-EGF domain converted $\mathrm{Vn}$ into a stronger DER activator (Vn:Spi EGF) (Fig. 1C). The $V n$ :Aos EGF chimera behaved as an inhibitor, rather than an activator and caused a reduction in ERK activation resulting from the ligand-independent activation of DER (Fig. 1D). These results show that the properties of $V n$ are changed when its EGF domain is swapped with that of Spi or Aos so that the chimeras behave like the factors from which the EGF domain is derived.

Vn:Spi EGF chimeras behave as strong activators

in vivo

In the embryo, ectopic activation of the DER pathway by sSpi using the Gal4-UAS system [Krüppel (Kr-gal4; UAS-sspi)] causes an expansion of ventral cell fates that can be monitored by expression of the ventral cell marker orthodenticle (otd) (Wie schaus et al. 1992; Brand and Perrimon 1993; Schweitzer et al. 1995b; Gabay et al. 1996)(Fig. 2D). Ectopic expression of native $\mathrm{Vn}$ ( $\mathrm{Kr}$-gal4; U AS-vn) caused no change in the expression of otd (Fig. 2B). The $\mathrm{Vn}: \mathrm{Vn}$ EGF chimera (Kr-gal4; UAS-vn:vn EGF) caused a very mild expansion of otd expression (Fig. 2C). This slight effect could be the result of higher expression of the transgene (cf. insets in Fig. 2, B and C). In contrast, ectopic expression of the $\mathrm{Vn}: S p i$ EGF chimera ( $\mathrm{Kr}$-gal4; U AS-vn:spi EGF) caused a dramatic expansion of otd expression that was similar to that seen with ectopic expression of sSpi (Fig. 2E,D).

In the wing, ectopic activation of the DER pathway is characterized by the appearance of extra veins (Sturtevant et al. 1993). Ectopic expression of native $V n$ in pupal interveins (1348-gal4; U A S-vn) produced a mild or mod- 


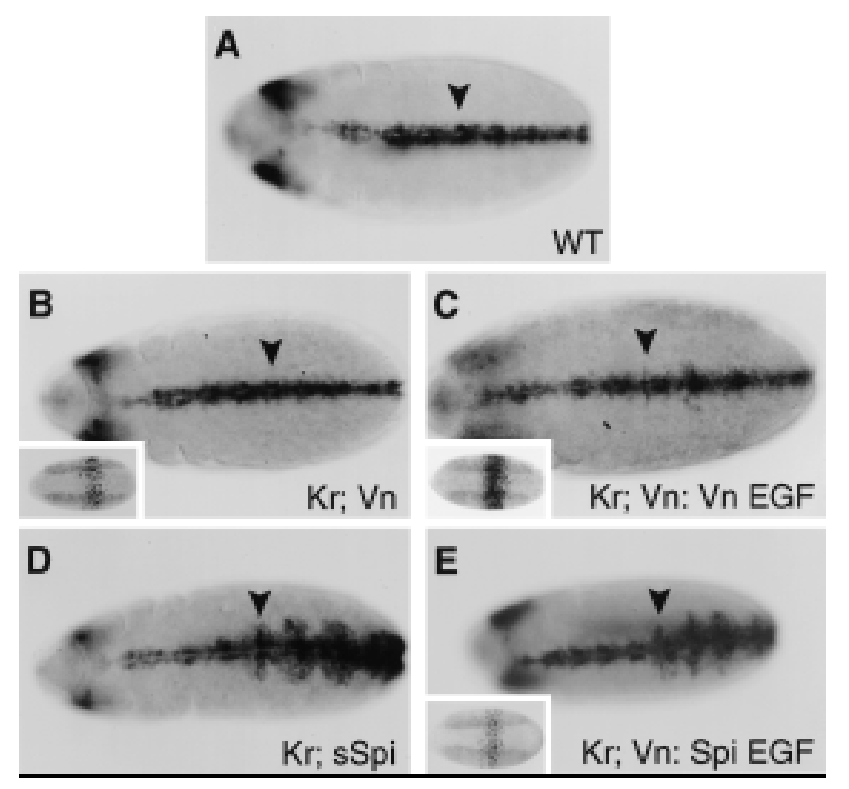

Figure 2. Ectopic expression of native and chimeric factors in the embryo. Each panel shows the expression of otd, which is a marker for ventral fates. (Insets in B, C, and E) Endogenous vn expression in two ventrolateral domains and ectopic expression of vn and the vn chimeras in a circumferential band spanning the $\mathrm{Kr}$ expression domain [second thoracic segment (T2)-fourth abdominal segment (A4)]. These controls show the level of ectopic expression of the factors. (A) Wild type. One to two rows of cells on either side of the ventral midline express otd. (B) $\mathrm{Kr}$-gal4; U AS-vn. There is no change in otd expression following ectopic expression of native $\mathrm{Vn}$ in the $\mathrm{Kr}$ domain. (C) $\mathrm{Kr}$ gal4; UAS-vn:vn EGF. There is a very mild expansion of otd expression following ectopic expression of the Vn:Vn EGF chimera. This may bethe result of higher ectopic expression of this factor than native $\mathrm{Vn}$ (cf. insets in $\mathrm{B}$ and $\mathrm{C}$ ). (D) $\mathrm{Kr}$-gal 4; U ASsspi. Ectopic expression of sSpi causes ventral cell fates to spread laterally in T2-A4 as seen by the expanded expression of otd. (E) Kr-gal4; UAS-vn:spi EGF. Ectopic expression of the $\mathrm{Vn}$ :Spi EGF chimera causes an expansion of otd expression similar to that seen after ectopic expression of native sSpi. Arrowheads in A-E indicate segment T2.

erate extra-vein phenotype, whereas ectopic expression of sSpi (1348-gal4; U AS-sspi) caused a strong extra-vein phenotype (Fig. 3G). A direct role for $\mathrm{Vn}$ in normal vein development has been shown; such a role has not been demonstrated for sSpi but is likely to be involved (Simcox et al. 1996; Schweitzer and Shilo 1997). Ectopic expression of the Vn:Vn EGF chimera (1348-gal4; UASvn:vn EGF) gave extra-vein phenotypes similar to those seen after ectopic expression of native $\mathrm{Vn}$ (Fig. 3F). In contrast, ectopic expression of the Vn:Spi EGF chimera (1348-gal4; UAS-vn:spi EGF) produced a strong extravein phenotype like that seen following ectopic expression of sSpi (Fig. 3H).

In the eye, ectopic activation of the DER pathway is characterized by loss of ommatidia, over-recruitment of cell types, and blistering (Baker and Rubin 1989; Freeman et al. 1992; Freeman 1996). Ectopic expression of native $\mathrm{Vn}$ posterior to the morphogenetic furrow in the eye disc (GMR-gal4; U AS-vn) had no effect on the adult eye phenotype (Fig. 4E), whereas, ectopic expression of sSpi (GMR-gal 4; UAS-sspi) and the Vn:Spi EGF chimera (GMR-gal4; UAS-vn:spi EGF) produced small disorganized eyes with blisters (Fig. 4G,H). Surprisingly ectopic expression of the Vn:Vn EGF chimera (GMR-gal4; UASvn:vn EGF) also showed a strong eye phenotype (Fig. 4F).

These in vivo data corroborate the biochemical data that $\mathrm{Vn}$ is a less potent activator of DER than sSpi. Additionally they show the EGF domain is a key feature that differentiates $\mathrm{Vn}$ and SSpi because $\mathrm{Vn}$ can be converted into a more potent DER activator if its EGF domain is swapped with that of Spi. The ability to differentially regulate signaling depending on whether $\mathrm{Vn}$ or sSpi is utilized may be one mechanism by which DER elicits specific cell responses during devel opment.
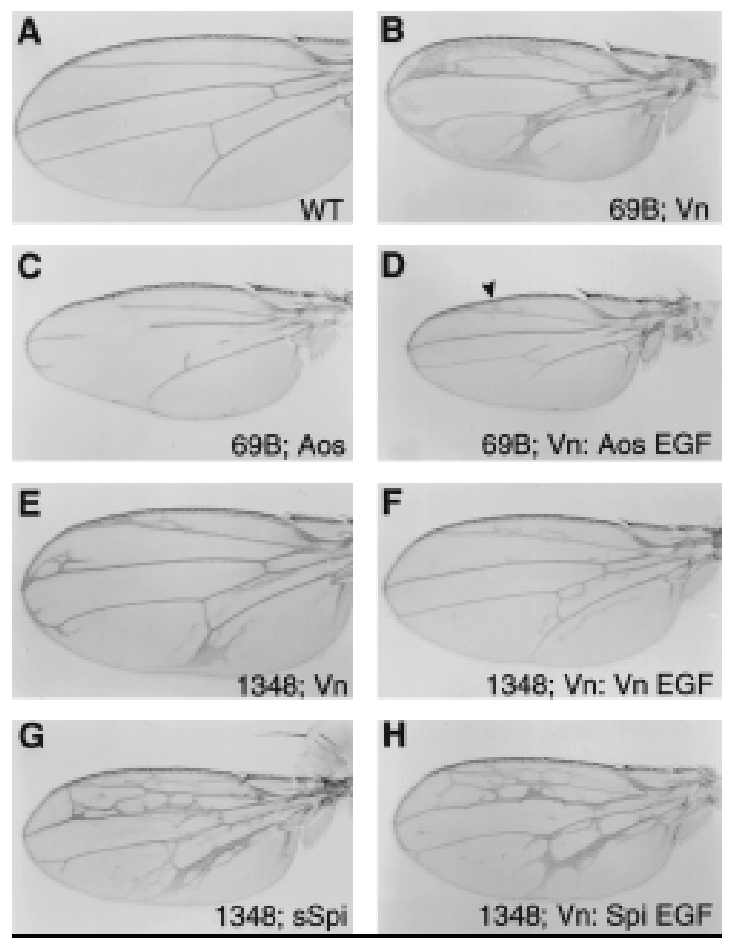

Figure 3. Ectopic expression of native and chimeric factors in the wing. (A) Wild-type wing showing the normal pattern of five longitudinal veins and two crossveins. (B) 69B-gal4; UAS-vn. Extra vein phenotype produced by overexpressing native $\mathrm{Vn}$ in the wing disc. The wing is smaller than wild type because vein cells that replace intervein cells are more compact. (C) 69Bgal 4; U AS-aos. (D) 69B-gal4; U AS-vn:aos EGF. Ectopic expression of native Aos (C) and the Vn:Aos EGF chimera (D) causes loss of wing veins and the wings are smaller than wild type due to a reduction in the number of vein and intervein cells. Ectopic expression of $\mathrm{Vn}$ :Aos EGF gives a milder vein loss phenotype than native Aos. The fusion between L1 (the margin) and L2 is unlikely to be due to expression of UAS-vn:aos EGF as it is al so seen in flies expressing the 69B-gal 4 gene al one (arrowhead in D). (E) 1348-gal4; U AS-vn. (F) 1348-gal4; U AS-vn:vn EGF. In E and $F$ there is a moderate extra-vein phenotype produced by ectopic expression of factors in pupal intervein regions. (G) 1348-gal 4; U AS-sspi. (H) 1348-gal 4; U AS-vn:spi EGF. In G and $\mathrm{H}$ there is a strong extra-vein phenotype produced by ectopic expression of sSpi and the Vn:Spi EGF chimera in pupal intervein regions. All wings are shown at the same magnification. 
EGF receptor activators and inhibitors
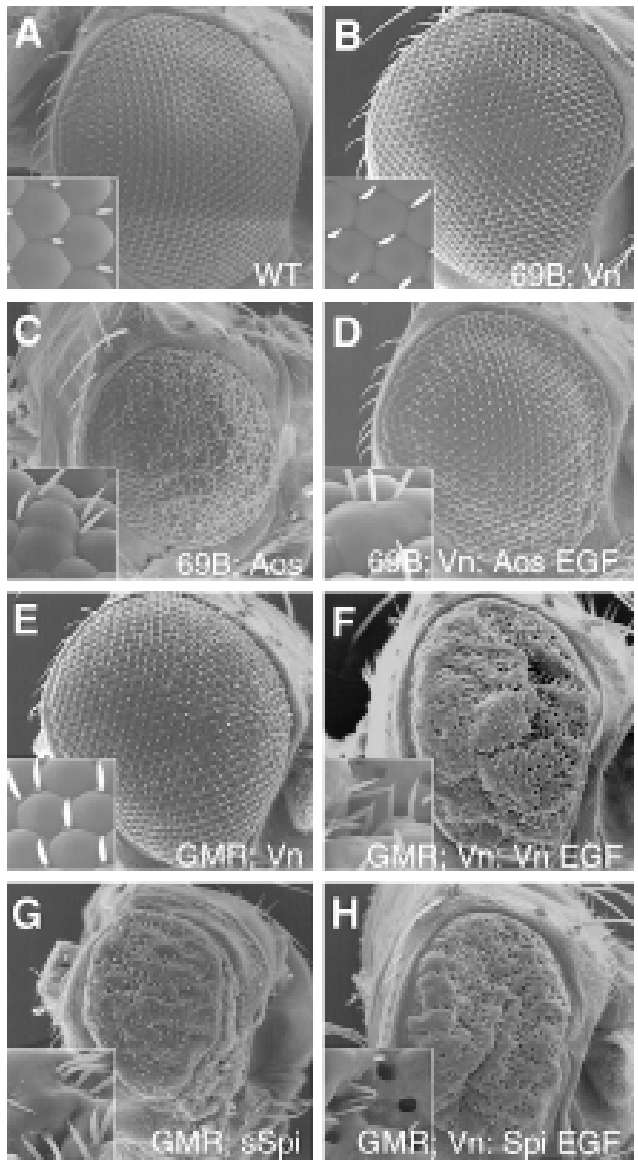

Figure 4. Ectopic expression of native and chimeric factors in the eye. All panels show scanning el ectron micrographs of adult eyes. (A) Wild-type eye showing the highly structured array of ommatidia, some of which are shown at higher magnification in the inset. (B) 69B-gal4; UAS-vn. Ectopic expression of native $\mathrm{Vn}$ in the eye disc produces eyes that are indistinguishable from wild type. (C) 69B-gal4; U AS-aos. (D) 69B-gal4; U AS-vn:aos EGF. (C,D) A rough eye phenotype caused by ectopic expression of native $A$ os and the $V n$ :Aos EGF chimera in the eye disc. The eyes are smaller than wild type and some ommatidia are fused (see insets). (E) GMR-gal4; UAS-vn. Overexpression of native $\mathrm{V} n$ in cells behind the morphogenetic furrow apparently has no effect. The occasional bristle duplications and depressions in the lenses are al so seen in eyes of flies expressing the GMR-gal 4 gene al one (not shown). (F) GMR-gal4;UAS-vn. (G) GMR-gal4; U AS-sspi. (H) GMR-gal4; U AS-vn:spi EGF. (F,G,H) Ectopic expression of factors produces disorganized eyes with loss of ommatidia and blisters. (A-H) Insets are fivefold magnification of a part of the whole eye image.

The Vn:Vn EGF chimera appears to be a more potent factor than native $V n$ in the eye because unlike ectopic Vn, which had no effect, ectopic Vn:Vn EGF produced a strong phenotype. This suggests that regions outsi de the EGF domain can affect the activity of a factor because the manipulation used to create the chimeras changed 4 residues flanking the EGF domain (Fig. 1A). This observation also underscores the importance of assaying for ligand function in a number of cell types because such modulating effects may only lead to appreciable differences in phenotype in some cell types.

\section{Vn:Aos EGF chimeras behave as inhibitors in vivo}

To test whether $\mathrm{Vn}$ could be converted into an inhibitor by swapping its EGF domain with that of Aos, we compared the effects of ectopic expression of $\mathrm{Vn}, \mathrm{Aos}$, and the $V n$ :Aos EGF chimera in larval wing and eye discs. $\mathrm{N}$ ative $\mathrm{Vn}$ produced an extra-vein phenotype when expressed ectopical ly in larval wing discs as expected for an activator of DER signaling (69B-gal4; U AS-vn; Fig. 3B). In the wing, ectopic suppression of the DER pathway is characterized by vein loss (Sawamoto et al. 1994; Schweitzer et al. 1995a) and ectopic expression of native Aos (69B-gal4; UAS-aos) or the Vn:Aos EGF chimera (69B-gal4; UAS-vn:aos EGF) resulted in vein loss (Fig. $3 C, D)$. The vein loss phenotype associated with ectopic expression of $V n$ :Aos EGF was not as severe as that caused by native Aos.

In the eye, reduction in activity of the DER pathway is characterized by loss of cell types and fusion of ommatidia (Clifford and Schüpbach 1989; Freeman 1994a). There was no observable effect on adult eye phenotype following ectopic expression of native $\mathrm{Vn}$ in eye discs (69B-gal4; UAS-vn), but ectopic expression of the Vn:Aos EGF chimera (69B-gal4; U AS-vn:aos EGF) produced a rough eye phenotype with fused lenses similar to, but not as severe as, that produced by ectopic expression of native Aos (69B-gal4; UAS-aos) (Fig. 4C,D). These results show that the EGF domain is a key determinant responsible for the difference between $\mathrm{Vn}$ and Aos and that the EGF swap is sufficient to convert a DER activator into an inhibitor. The Vn:Aos EGF chimera is apparently not as potent an inhibitor as native Aos in the eye or the wing, suggesting that other regions of the proteins ( $\mathrm{n}$ and/or Aos) may play modulating roles.

\section{Concluding remarks}

We have shown that $\mathrm{Vn}$ is a moderate activator of DER signaling and can be converted into a strong DER activator by exchanging the Vn EGF domain with that of Spi or into an inhibitor by exchanging the Vn EGF domain with that of Aos. This demonstrates that the EGF domain is the key feature that gives each ligand its distinct property. The result is important for understanding the function of the novel extracellular inhibitor Aos, as it suggests that the EGF domain is sufficient for DER inhibition while other regions of the protein are not essential (but may play modulating roles). There is currently no definitive evidence that Aos elicits its effect on DER signaling by binding DER directly; however, our results suggest that the EGF domain is a critical region for mediating the effect.

The fly system, which can efficiently test the function of chimeras in vivo, may be a powerful tool for precisely defining the region within the EGF domain that is characteristic of an inhibitor. Human EGF chimeras with substitutions or insertions of sequence from the B loop of the Aos EGF domain functioned as agonists rather than antagonists (van de Poll et al. 1997). N everthel ess, these results demonstrate that fly/vertebrate chimeras are able to bind and elicit a response from a vertebrate EGF re- 
ceptor and suggest that inhibitory sequences identified in the fly system could be used to modify vertebrate factors to function as antagonists. The development of ErbB antagonists has significant clinical implications, as these receptors and their ligands function in growth of tumor cells (Groenen et al. 1994).

\section{Materials and methods}

Generation of chimeric genes

The chimeras were made by combining a vn cDNA lacking the EGF motif with fragments encoding the EGF motif of vn, spi, or aos. To generate the cDN A lacking the EGF motif, a vn type 1 CDN A corresponding to nucleotides 1679-3980 was used as a template to amplify 5' and $3^{\prime}$ regions extending to 10 amino acids before the first cysteine and from 10 amino acids after the sixth cysteine, respectively. For the $5^{\prime}$ fragment, the primers 5'-ATTAACCCTCACTAAAG-3' (this corresponds to the T3 region in pBS, the vector in which the vn CDNA is inserted) and $5^{\prime}-A C$ CCGGGAAAGTGAACTGGTGAGGCCTTG-3', which incorporates an Xmal site at the $3^{\prime}$ end, were used. For the $3^{\prime}$ fragment the primers 5'-GACTAGTGTTGCAATCTACGGCCAAATAC-3', which incorporates the Spel site at the $3^{\prime}$ end, and 5'-AATACGACTCACTATAG-3' ( 77 region in pBS) were used. Clones encoding the EGF motif from vn, spi, and aos were also generated by PCR amplification and incorporated 5' Xmal and 3' Spel sites for insertion into the vn CDN A lacking the EGF motif. The primers to amplify the vn EGF motif from a vn CDNA were 5'-ACCCGGGCCCACGGACCGGTCAGCCTCG-3' and 5'-GACTAGTAAAATATCTACTGTCGGGCC-3'. The primers used to amplify the spi EGF domain from genomic DNA were 5'-ACCCGGGAGGCCCAATATTACATTCCCC-3' and 5'-GACTAGTCAGGTAAGTATTGTCGATCTC $-3^{\prime}$. The primers used to amplify the aos EGF domain from an aos CDNA (kindly provided by K. Sawamoto, University of Tokyo, Japan) were 5'-ACCCGGGGACAGTCCGGGCTACAGATATC-3' and 5'-GACTAGTTATCACGCCGGATTGCGTGTG-3'. All reactions contained $100 \mathrm{ng}$ of template, 10 pmoles of each primer, $20 \mathrm{~mm}$ Tris- $\mathrm{HCl}(\mathrm{pH}$ 8.4), $50 \mathrm{~mm} \mathrm{KCl}, 5 \mathrm{~mm} \mathrm{MgCl}, 2 \mathrm{~mm}$ dNTPs, and 5 units of Taq DNA polymerase (GIBCO BRL). Cycling conditions were $1 \mathrm{cycle}$ at $95^{\circ} \mathrm{C}$ for $5 \mathrm{~min}$, followed by $30 \mathrm{cycles}$ at $95^{\circ} \mathrm{C}$ for $1 \mathrm{~min}, 60^{\circ} \mathrm{C}$ for $1 \mathrm{~min}$, and $72^{\circ} \mathrm{C}$ for 1.5 $\mathrm{min}$. For in vitro studies, CDN As encoding native $\mathrm{Vn}$ and the $\mathrm{Vn}$ chimeras were cloned into the Xhol and BamHI sites of pM K33 (Schnepp et al. 1996). For in vivo studies, the genes were cloned into the Xhol and Xbal sites of the P-element vector pUAST (Brand and Perrimon 1993).

In vitro assays of DER signaling

Stable Drosophila S2 cell lines were established that express and secrete the $V n$ and the $V n$ chimeric proteins $(V n, V n: V n$ EGF, Vn:Spi EGF, and Vn:A os EGF) (Schnepp et al. 1996). Receptor phosphorylation assays were performed according to Schweitzer et al. (1995b): M edium collected from stably transfected $\mathrm{S} 2$ cells expressing native $\mathrm{Vn}$, native $\mathrm{sSpi}$, or the $\mathrm{Vn}$ chimeric proteins was added to a monolayer of $\mathrm{S} 2$ cells expressing DER. After a 5-min incubation, DER was immunoprecipitated from the cells and samples were run on duplicate SDS-polyacrylamide gels and blotted. One blot was probed with anti-phosphotyrosine antibodies (kindly provided by M. Coggeshall, The Ohio State University) to show the level of DER tyrosine phosphorylation, and one blot was probed with anti-DER antibodies to show that the amount of protein loaded in each lane was similar. The relative amounts of $\mathrm{Vn}$ and the $\mathrm{Vn}$ chimeras were determined using an anti-Vn antibody (Schnepp et al. 1996). For the doseresponse experiment, $\mathrm{Vn}$-conditioned serum-free medium was concentrated $60 \times$ by centrifugation through a Millipore Biomax $10 \mathrm{~K}$ column. The concentrate was subsequently diluted over an eightfold range with 0.125 increments. ERK assays were performed according to Gabay et al. (1997): Conditioned medium was added to S2-DER cells for $14 \mathrm{~min}$. The cells were lysed, and samples were run on dupl icate gels and blotted. One blot was probed with anti-diphosphorylated ERK (Sigma M 8159) to show the level of ERK phosphorylation, and one blot was probed with anti-ERK (Sigma M-5670) to show that the amount of protein loaded in each lane was similar. To show the ligand-independent inhibition by Vn:Aos EGF, S2-DER cells were incubated with Vn:Aos EGF-conditioned medium for $14 \mathrm{~min}$. Controls were incubated with medium from untransfected S2 cells.

Transgenic cell lines

Multiple transgenic lines were established for each construct: 8 lines of
UAS-vn; 6 lines of U AS-vn:vn EGF; 17 lines of U AS-vn:vn spi EGF; and 12 lines of U AS-vn:aos EGF. Single transgenic lines of UAS-aos (kindly provided by R. Howes and M. Freeman, M RC, Cambridge, UK) and U ASsspi were examined.

Ectopic expression in the embryo

To examine the effect of ectopic expression of the factors in the embryo the U AS transgenes were expressed with the $\mathrm{Kr}$-gal 4 driver (ectopic expression in segments T2-A4) and otd expression was monitored. otd expression serves as a marker for ventral cell fate. Embryos were collected for $2 \mathrm{hr}$ from a cross between $\mathrm{Kr}$-gal 4/TM 3 flies and flies with the native or chimeric EGF-like genes (U AS-vn, U AS-vn:vn EGF, U AS-sspi, or UAS-vn:spi EGF). The embryos were aged for $5.5 \mathrm{hr}$ at $25^{\circ} \mathrm{C}$ (about stage 11) and hybridized with a digoxygenin-labeled otd antisense RN A probe. In situ hybridization to young embryos (1-3 hr) with a vn probe was used to screen for lines in which vn and the vn chimeras were ectopically expressed in the $\mathrm{Kr}$ pattern at similar levels. Lines expressing comparable levels of vn and vn:spi EGF were found. The line with the lowest expression of vn:vn EGF was used, but it had a slightly higher expression level than vn and vn:spi EGF.

Ectopic expression in the wing

To analyze the effect of ectopic expression of the factors in the wing the UAS transgenes were expressed with the 1348 -gal 4 or $69 \mathrm{~B}$-gal 4 drivers, and the venation pattern was examined $\left(25^{\circ} \mathrm{C}\right)$. When ectopically expressed in pupal intervein regions (1348-gal4) UAS-vn produced a mild (4 lines) or moderate (4 lines, Fig. 3E) extra-vein phenotype; U AS-vn:vnEGF produced a mild (2 lines), moderate (2 lines, Fig. 3F), or strong (2 lines) extra-vein phenotype; U AS-sspi produced a strong extra-vein phenotype (1 line, Fig. 3G); and U AS-vn:spi-EGF produced a moderate (6 lines) or strong (11 lines, Fig. 3H) extra-vein phenotype. We used the 69B-gal 4 driver, which causes ectopic expression in the larval discs, to test the effect of ectopic expression of UAS-aos and the UAS-vn:aosEGF chimera in the wing because these transgenes did not produce a phenotype when ectopically expressed with the 1348-gal 4 driver. When ectopically expressed in the wing UAS-aos (1 line that shows strong expression, Fig. $3 \mathrm{C}$ ) and U AS-vn:aos-EGF (12 lines that showed similar phenotypes, shown in Fig. 3D) caused loss of veins. M ost 69B-gal 4; U ASvn animals die, but a few adults survived and these had a strong extravein phenotype (Fig. 3B).

Ectopic expression in the eye

To analyze the effect of ectopic expression of the factors in the eye the transgenes were expressed with the GMR-gal $4\left(17^{\circ} \mathrm{C}\right)$ or $69 \mathrm{~B}$-gal $4\left(25^{\circ} \mathrm{C}\right)$ drivers, and the adult eyes were examined. Eye defects were visible with the light microscope for flies expressing U AS-vn:vn EGF (6 lines tested, 1 line showed a weak phenotype), U AS-vn:spi EGF (8 lines tested), and UAS-sspi (1 line tested). No defects were seen following expression of U AS-vn with GMR-gal 4 (2 lines tested) or 69B-gal 4 (8 lines tested). We used the 69B-gal 4 driver to determine the effect of ectopic expression of U AS-aos (1 line) and U AS-vn:aos EGF (12 lines) because the latter did not produce a phenotype when ectopical ly expressed with GMR-gal 4. A rough phenotype was seen in all lines tested. For SEM analysis of eyes by scanning electron microscopy, one representative line was examined for each construct. Fly heads were dehydrated through an ethanol series (25\%, 50\%, 75\%, $2 \times 100 \%)$, followed by $50 \%$ hexamethyldisilazane (HMDS) in ethanol and $2 \times 100 \%$ HMDS for $2 \mathrm{hr}$ each. The heads were air-dried overnight, sputter coated with gold/palladium, and analyzed with a Phillips XL30 scanning electron microscope at $20.0 \mathrm{kV}$.

\section{Acknowledgments}

We thank M. Coggeshall, M. Freeman, R. Howes, K. Sawamoto, R. Finkel stein and the Indiana Stock Center for reagents, and $C$. Beall and $E$. Gottlieb for critical reading of the manuscript. This work was supported by the $\mathrm{N}$ ational Science Foundation (grant $97-24078$ to A.S.) and an Ohio State University Alumni Research Award (to B.S.).

The publication costs of this article were defrayed in part by payment of page charges. This article must therefore be hereby marked "advertisement" in accordance with 18 USC section 1734 solely to indicate this fact.

\section{References}

Baker, N.E. and G.M. Rubin. 1989. Effect on eye development of domi- 
nant mutations in Drosophila homologue of the EGF receptor. $\mathrm{Na}$ ture 340: 150-153.

Brand, A. and N. Perrimon. 1993. Targeted gene expression as a means of altering cell fates and generating dominant phenotypes. Development 118: 401-415.

Clifford, R.T. and T. Schüpbach. 1989. Coordinately and differentially mutable activities of torpedo, the Drosophila melanogaster homolog of the vertebrate EGF receptor gene. Genetics 122: 771-787.

Egan, S.E. and R.A. Weinberg. 1993. The pathway to signal achievement. Nature 365: 781-783.

Freeman, M. 1994a. Misexpression of the Drosophila argos gene, a se creted regulator of cell determination. Development 120: 2297-2304.

- - . 1994b. The spitz gene is required for photoreceptor determination in the Drosophila eye where it interacts with the EGF receptor. Mech. Dev. 48: 25-33.

-. 1996. Reiterative use of the EGF receptor triggers differentiation of all cell types in the Drosophila eye. Cell 87: 651-660.

Freeman, M., C. Klämbt, C.S. Goodman, and G.M. Rubin. 1992. The argos gene encodes a diffusible factor that regulates cell fate in the Drosophila eye. Cell 69: 963-975.

Gabay, L., H. Scholz, M. Golembo, A. Klaes, B.-Z. Shilo, and C. Klämbt. 1996. EGF receptor signaling induces pointed P1 transcription and inactivates $Y$ an protein in the Drosophila embryonic ventral ectoderm. Development 122: 3355-3362.

Gabay, L., R. Seger, and B.-Z. Shilo. 1997. In situ activation pattern of Drosophila EGF receptor pathway during development. Science $\mathbf{2 7 7}$ 1103-1106.

Golembo, M., R. Schweitzer, M. Freeman, and B.-Z. Shilo. 1996. argos transcription is induced by the Drosophila EGF receptor pathway to form an inhibitory feedback loop. Development 122: 223-230.

González-Reyes, A., H. Elliott, and D. St Johnston. 1995. Polarization of both major body axes in Drosophila by gurken-torpedo signaling. Nature 375: 654-658.

Groenen, L.C., E.C. Nice, and A.W. Burgess. 1994. Structure-function relationships for the EGF/TGF- $\alpha$ family of mitogens. Growth Factors 11: $235-257$.

Neuman-Silberberg, F. and T. Schüpbach. 1993. The Drosophila gene gurken produces a dorsally localized RNA and encodes a TGF $\alpha$-like protein. Cell 75: 165-174.

Perrimon, N . and L.A. Perkins. 1997. There must be 50 ways to rule the signal: The case of the Drosophila EGF receptor. Cell 89: 13-16.

Rutledge, B.J., K. Zhang, E. Bier, Y.N. Jan, and N. Perrimon. 1992. The Drosophila spitz gene encodes a putative EGF-like growth factor involved in dorsal-ventral axis formation and neurogenesis. Genes \& Dev. 6: 1503-1517.

Sawamoto, K., H. Okano, Y. Kobayakawa, S. Hayashi, K. Mikoshiba, and T. Tanimura. 1994. The function of argos in regulating cell fate decisions during Drosophila eye and wing vein devel opment. Dev. Biol. 164: 267-276.

Schnepp, B., G. Grumbling, T. Donaldson, and A. Simcox. 1996. Vein is a novel component in the Drosophila epidermal growth factor receptor pathway with similarity to the neuregulins. Genes \& Dev. 10: 2302-2313.

Schweitzer, R. and B.-Z. Shilo. 1997. A thousand and one roles for the Drosophila EGF receptor. Trends Genet. 13: 191-196.

Schweitzer, R., R. Howes, R. Smith, B.-Z. Shilo, and M. Freeman. 1995a. Inhibition of Drosophila EGF receptor activation by the secreted protein Argos. Nature 376: 699-702.

Schweitzer, R., M. Shaharabany, R. Seger, and B.-Z. Shilo. 1995b. Se creted spitz triggers the DER signaling pathway and is a limiting component in embryonic ventral ectoderm determination. Genes \& Dev. 9: 1518-1529.

Simcox, A. 1997. Differential requirement for EGF-like ligands in Drosophila wing development. Mech. Dev. 62: 41-50.

Simcox, A., G. Grumbling, B. Schnepp, C. Bennington-M athias, E. Hersperger, and A. Shearn. 1996. Molecular, phenotypic, and expression analysis of vein, a gene required for growth of the Drosophila wing disc. Dev. Biol. 177: 475-489.

Sturtevant, M.A., M. Roark, and E. Bier. 1993. The Drosophila rhomboid gene mediates the localized formation of wing veins and interacts genetically with components of the EGF-R signaling pathway. Genes \& Dev. 7: 961-973.

van de Poll, M.L.M., M.J.H. van Vugt, A.E.G. Lenferink, and E.J.J. van
Zoelen. 1997. Insertion of Argos sequences into the B-loop of epidermal growth factor results in a low-affinity ligand with strong agonistic activity. Biochemistry 36: 7425-7431.

Wieschaus, E., N. Perrimon, and R. Finkelstein. 1992. Orthodenticle activity is required for the development of medial structures in the larval and adult epidermis of Drosophila. Devel opment 115: 801-811.

Yarnitzky, T., L. Min, and T. Volk. 1997. The Drosophila neuregulin homolog Vein mediates inductive interactions between myotubes and their epidermal attachment cells. Genes \& Dev. 11: 2691-2700. 


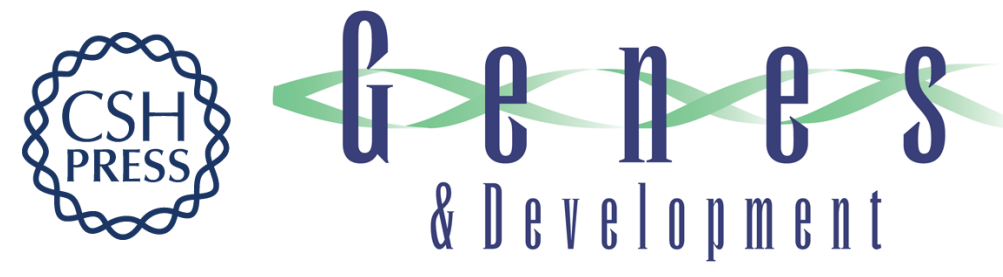

\section{EGF domain swap converts a Drosophila EGF receptor activator into an inhibitor}

Bruce Schnepp, Timothy Donaldson, Gary Grumbling, et al.

Genes Dev. 1998, 12:

References This article cites 27 articles, 11 of which can be accessed free at:

http://genesdev.cshlp.org/content/12/7/908.full.html\#ref-list-1

License

Email Alerting Receive free email alerts when new articles cite this article - sign up in the box at the top Service right corner of the article or click here.

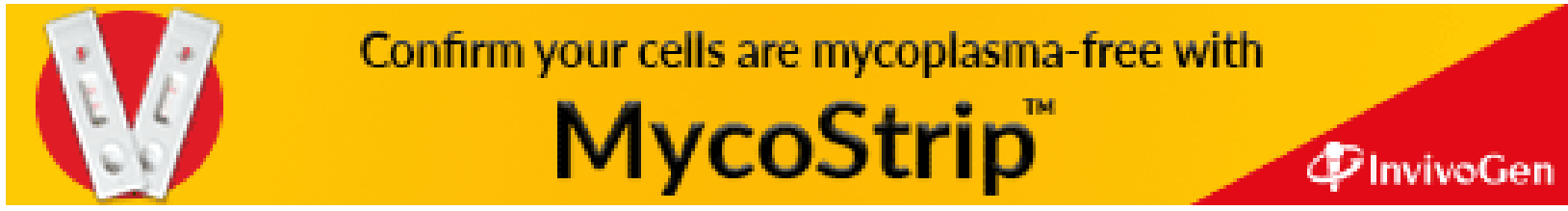

